\title{
STABILITY ROBUSTNESS IN CLOSED LOOP VIBRATIONAL CONTROL
}

\author{
P. T. KABAMBA ${ }^{1}$, S. M. MEERKOV ${ }^{2 *}$ AND E.-K. POH ${ }^{2}$ \\ ${ }^{1}$ Department of Aerospace Engineering, The University of Michigan, Ann Arbor, MI 48109-2140, U.S.A \\ ${ }^{2}$ Department of Electrical Engineering and Computer Science, The University of Michigan, Ann Arbor, MI 48109-2122, U.S.A
}

\section{SUMMARY}

In this paper, we consider the robust stability analysis and synthesis problems for closed-loop vibrational control. In the analysis problem, we derive an upper bound on the allowable unstructured uncertainty which preserves the stability of a closed-loop vibrationally stabilized system. In the synthesis problem, we establish a necessary and sufficient condition for the existence of a single vibrational controller that stabilizes a polytope of plants. (C) 1998 John Wiley \& Sons, Ltd.

Key words: vibrational control; parametric uncertainty

\section{INTRODUCTION AND PROBLEM FORMULATION}

Consider the system

$$
\begin{gathered}
\dot{x}=A x+B u f\left(\begin{array}{l}
t \\
\varepsilon \\
\varepsilon
\end{array}\right) \\
f(t)=f(t+T), \quad T \neq 0, \quad \frac{1}{T} \int_{0}^{T} f(t) \mathrm{d} t=0 \\
\phi(t)=\int_{0}^{t} f(\tau) \mathrm{d} \tau, \quad \frac{1}{T} \int_{0}^{T} \phi(t) \mathrm{d} t=0
\end{gathered}
$$

where $x \in \mathbb{R}^{n}, u \in \mathbb{R}, A$ and $B$ are constant matrices of appropriate dimensions, $f(t)$ is a piecewise continuous function, and $\varepsilon$ is a small positive parameter, $0<\varepsilon \ll 1$. A characteristic feature of this system is that the control, $u$, enters the open-loop system dynamics as an amplitude of a periodic, zero average function, and this amplitude can be chosen to depend on the system state vector $x$. Such systems belong to a large class of bilinear systems found in mechanical systems. ${ }^{1}$ Another example is the periodic operation of chemical reactors ${ }^{2}$ where the input flow vibrations are introduced so that the closed-loop system behaves as desired.

This paper was recommended for publication by editor A. Sideris

\footnotetext{
* Correspondence to: S. M. Meerkov, Department of Electrical Engineering and Computer Science, The University of Michigan, Ann Arbor, MI 48109-2122, U.S.A.
}

Contract/grant sponsor: U.S. Army Research Office

Contract/grant number: DAAL03-90-G-0004

CCC 1049-8923/98/121101-11\$17.50

(C) 1998 John Wiley \& Sons, Ltd. 
A control theory for system (1), referred to as closed-loop vibrational control, has been developed in References 3 and 4. In particular, the following has been proved:

\section{Theorem $1.1^{3,4}$}

There exists a matrix $K$ and a sufficiently small positive $\varepsilon_{0}$ such that for all $0<\varepsilon \leqslant \varepsilon_{0}$ system (1) with state-space feedback

$$
u=\frac{K}{\varepsilon} x=\frac{1}{\varepsilon}\left[k_{n} k_{n-1} \cdots k_{2} k_{1}\right] x
$$

is asymptotically stable if and only if $(A, B)$ is stabilizable and the sum of all the controllable eigenvalues of $A$ is negative.

The present paper is devoted to the property of stability robustness in closed-loop vibrational control. It is assumed that (1) is the nominal plant, whereas the true plant is defined by

$$
\dot{x}=(A+\Delta A) x+B u f\left(\begin{array}{l}
t \\
\varepsilon
\end{array}\right)
$$

where $\Delta A$ is a constant perturbation matrix with no time delay. In this paper, we examine the analysis and synthesis problems arising out of uncertainties in the system matrix $A$ and ignore actuator imperfections, i.e. the matrix $B$ is assumed to be known exactly and does not contain any variations.

In the analysis problem, Section 2 gives a bound on the spectral radius of $\Delta A$ so that for all $\Delta A$ 's that meet this bound, a controller (4), which stabilizes the nominal plant (1), also stabilizes the perturbed system (5). Assuming that the characteristic polynomial of (5) belongs to a polytope, Section 3 gives a condition which guarantees the existence of a controller (4) such that the closed-loop system (5), (4) is asymptotically stable for all members of the polytope. The conclusions are formulated in Section 4.

The following notations are used. The transpose of a matrix $A$ is denoted as $A^{\mathrm{T}}$. We denote the maximum singular value, maximum eigenvalue and minimum eigenvalue of $A$ by $\sigma_{\max }(A)$, $\lambda_{\max }(A)$, and $\lambda_{\min }(A)$, respectively. $\operatorname{Tr} A$ denotes the trace of matrix $A$. For a periodic function $\beta(t)$ with period $T, \overline{\beta(t)}=1 / T \int_{0}^{T} \beta(t) \mathrm{d} t$. To satisfy the conditions of Theorem 1.1 , we assume throughout this paper that the pair $(A, B)$ in (1) is controllable and $\operatorname{Tr} A$ is negative.

\section{ANALYSIS}

Consider system (5) with feedback (4)

$$
\dot{x}=\left(A+\Delta A+B \frac{K}{\varepsilon} f\left(\frac{t}{\varepsilon}\right)\right) x
$$

\section{Theorem 2.1}

Assume that the matrix $K$ is chosen so that for all $0<\varepsilon \leqslant \varepsilon_{1}$ system (6) with $\Delta A=0$ is asymptotically stable. Then there exists a sufficiently small positive $\varepsilon_{0} \leqslant \varepsilon_{1}$ such that for all $0<\varepsilon \leqslant \varepsilon_{0}$ system (6) with $\Delta A \neq 0$ is asymptotically stable if

$$
\sigma_{\max }\left(\overline{\Phi^{-1}\left(\frac{t}{\varepsilon}\right) \Delta A \Phi\left(\frac{t}{\varepsilon}\right)}\right) \leqslant \frac{\lambda_{\min }(Q)}{\lambda_{\max }(P)}
$$


where $\Phi(t)$ is a state transition matrix for $\dot{x}=B K f(t) x, P$ is the unique positive-definite solution of the Lyapunov equation

$$
P \bar{A}+\bar{A}^{\mathrm{T}} P+2 Q=0
$$

$Q$ is some positive-definite matrix, and

$$
\bar{A}=\overline{\Phi^{-1}\left(\begin{array}{l}
t \\
\varepsilon
\end{array}\right) A \Phi\left(\frac{t}{\varepsilon}\right)}
$$

Proof. Define

$$
x(t)=\Phi\left(\begin{array}{l}
t \\
\varepsilon
\end{array}\right) \zeta(t)
$$

and rewrite (6) as

$$
\dot{\zeta}=\Phi^{-1}\left(\begin{array}{l}
t \\
\varepsilon
\end{array}\right)(A+\Delta A) \Phi\left(\frac{t}{\varepsilon}\right) \zeta
$$

In time $\tau=t / \varepsilon$, this is an equation in the standard form. ${ }^{5}$ Therefore, applying the averaging principle and taking into account (2), we obtain the following averaged equation: ${ }^{6}$

$$
\dot{\bar{\zeta}}=\overline{\Phi^{-1}\left(\begin{array}{l}
t \\
-w \\
\varepsilon
\end{array}(A+\Delta A) \Phi\left(\frac{t}{\varepsilon}\right)\right.} \bar{\zeta}=(\bar{A}+\overline{\Delta A}) \bar{\zeta}
$$

where

$$
\overline{\Delta A}=\overline{\Phi^{-1}\left(\begin{array}{l}
t \\
\frac{c}{\varepsilon}
\end{array}\right) \Delta A \Phi\left(\frac{t}{\varepsilon}\right)}
$$

Note that the last equality in (12) is obtained due to the linearity of the averaging operation. From Reference 7, we know that the averaged equation (12) is stable if

$$
\sigma_{\max }(\overline{\Delta A}) \leqslant \frac{\lambda_{\min }(Q)}{\lambda_{\max }(P)}
$$

where $P$ is the unique matrix that satisfies the Lyapunov equation (8) and $Q$ is any positive-definite matrix.

Hence, for each $\Delta A$ satisfying (14), the averaged equation (12) is asymptotically stable, and there exists $\varepsilon_{\Delta A}$ such that for all $0<\varepsilon \leqslant \varepsilon_{\Delta A}$, system (11) is also asymptotically stable. ${ }^{5-7}$ Due to (10) asymptotic stability of (11) implies also the asymptotic stability of (6). In Reference 8, it was shown that $\varepsilon_{\Delta A}$ is a continuous function of the elements of the matrix $\Delta A$. Since the set of $\Delta A$ satisfying (14) is closed and bounded, it follows that there exists a uniform lower bound for $\varepsilon_{\Delta A}$. The proof is completed by setting $\varepsilon_{0}=\min _{\Delta A} \varepsilon_{\Delta A}$.

\section{Remark 2.1}

As it follows from Reference 7, the largest robustness bound in (7), is attained when $Q$ of (8) is selected as an identity matrix. 
The perturbation bound, given in (7), is imposed on $\Delta A$ in an indirect manner, through the averaged perturbed matrix $\overline{\Delta A}$. However, when $A$ and $B$ are in the controllable canonical form, the robustness bound can be re-formulated directly in terms of the spectral radius of $\Delta A$. This is carried out below. Indeed, assume that $A$ and $B$ are in the controllable canonical form. Then the state transition matrix $\Phi(\tau)$ is given by ${ }^{4}$

$$
\Phi(\tau)=\left[\begin{array}{cccc}
1 & \cdots & 0 & 0 \\
\vdots & & \ddots & \vdots \\
0 & \cdots & 1 & 0 \\
\frac{k_{n}}{k_{1}}\left(\mathrm{e}^{k_{1} \phi(\tau)}-1\right) & \cdots & \frac{k_{2}}{k_{1}}\left(\mathrm{e}^{k_{1} \phi(\tau)}-1\right) & \mathrm{e}^{k_{1} \phi(\tau)}
\end{array}\right]
$$

Note that the state transition matrix $\Phi(\tau)$ remains well defined for $k_{1}=0$. Since $\operatorname{Tr} A$ is negative and $\overline{\phi(\tau)}=0$, we select $k_{1}=0$ so that (13) can be simplified into the following form:

$$
\overline{\Delta A}=\Delta A+\Delta A_{1}
$$

where

$$
\Delta A_{1}=\left[\begin{array}{cccc}
1 & \cdots & 0 & 0 \\
\vdots & & \ddots & \vdots \\
0 & \cdots & 1 & 0 \\
k_{n} \sum_{i=1}^{n-1} \Delta A_{i n} k_{n-i+1} \overline{\phi^{2}} & \ldots & k_{2} \sum_{i=1}^{n-1} \Delta A_{i n} k_{n-i+1} \overline{\phi^{2}} & 0
\end{array}\right]
$$

and $\Delta A_{i j}$ denotes the $(i, j)$ th element of the perturbation matrix $\Delta A$.

\section{Corollary 2.1}

Assume that $A$ and $B$ are in controllable canonical form and $k_{1}$ in (4) is 0 . Then there exists a positive $\varepsilon_{0}$ sufficiently small such that for all $0<\varepsilon \leqslant \varepsilon_{0}$, the system (6) is stable if

$$
\sigma_{\max }(\Delta A)+\sigma_{\max }\left(\Delta A_{1}\right) \leqslant \frac{1}{\lambda_{\max }(P)}
$$

where $P$ is the solution of (8) with $Q=I$.

Proof. The proof follows directly from Theorem 2.1, (16) and the fact that $\sigma_{\max }\left(\Delta A+\Delta A_{1}\right)$ $\leqslant \sigma_{\max }(\Delta A)+\sigma_{\max }\left(\Delta A_{1}\right)$.

\section{Remark 2.2}

The value of $\varepsilon_{0}$, mentioned in Theorems 1.1 and 2.1, can be estimated using the method described in Reference 8. As it is usual in asymptotic analysis, this estimate is extremely conservative. In practice, however, much larger $\varepsilon$ 's ensure the correspondence between the original and the averaged equation. It was also numerically shown in Reference 8 that for systems with eigenvalues with magnitude of the order 1 , the value of $\varepsilon_{0} \simeq 0 \cdot 1$ is sufficient for stability of the averaged equation to imply the stability of the original equation. 


\section{Example 2.1}

Consider the system (6) with

$$
A=\left[\begin{array}{rr}
0 & 1 \\
0 & -3
\end{array}\right], \quad B=\left[\begin{array}{l}
0 \\
1
\end{array}\right], \quad f(\tau)=\sin (\tau)
$$

and $K=\left[\begin{array}{ll}2 & 0\end{array}\right]$. Applying the averaging principle, we obtain the averaged equation (12) with

$$
\bar{A}=\left[\begin{array}{rr}
0 & 1 \\
-2 & -3
\end{array}\right]
$$

From (18), the allowable range of the perturbation matrix which ensures the asymptotic stability of the original system (6) is given by

$$
\sigma_{\max }(\Delta A)+2\left|\Delta A_{12}\right| \leqslant 0 \cdot 382
$$

\section{SYNTHESIS}

Consider a system described by differential equation of the order $n$

$$
x^{(n)}+\left(a_{10}+\Delta a_{10}\right) x^{(n-1)}+\cdots+\left(a_{n 0}+\Delta a_{n 0}\right)=u f(t / \varepsilon)
$$

where $a_{i}, i=1, \ldots, n$ denote the nominal $i$ th coefficient of the differential equation (19) and $\Delta a_{i}$, $i=1, \ldots, n$ the corresponding perturbation in the $i$ th coefficient. We assume that the characteristic polynomial of the differential equation (19) belongs to the class $\mathscr{P}$ defined as follows:

$$
\mathscr{P}=\left\{p(s)=\sum_{j=1}^{m} \alpha_{j} p_{j}(s): \alpha_{j} \geqslant 0, j=1, \ldots, m ; \sum_{j=1}^{m} \alpha_{j}=1\right\}
$$

Here

$$
p_{j}(s)=s^{n}+a_{1}^{j} s^{n-1}+\cdots+a_{n}^{j}, \quad j=1, \ldots, m
$$

are the vertex polynomials and $a_{i}^{j}, i=1, \ldots, n$ denote the $i$ th coefficient of the $j$ th vertex polynomial.

Equivalently, the system (19) can be expressed in the form of (5) with $A$ and $B$ in the controllable canonical form and the perturbation matrix $\Delta A$ containing the terms $\Delta a_{i}$, $i=1, \ldots, n$ in the last row

$$
\begin{gathered}
A=\left[\begin{array}{cccc}
0 & 1 & \cdots & 0 \\
\vdots & & \ddots & \vdots \\
0 & 0 & \cdots & 1 \\
-a_{n 0} & -a_{(n-1) 0} & \cdots & -a_{10}
\end{array}\right], \quad B=\left[\begin{array}{c}
0 \\
\vdots \\
0 \\
1
\end{array}\right], \\
\Delta A=\left[\begin{array}{cccc}
0 & 0 & \cdots & 0 \\
\vdots & & \ddots & \vdots \\
0 & 0 & \cdots & 0 \\
-\Delta a_{n 0} & -\Delta a_{(n-1) 0} & \cdots & -\Delta a_{10}
\end{array}\right]
\end{gathered}
$$


The following results gives condition on the existence of a stabilising gain matrix $K$ for such a perturbed system (5) with characteristic polynomial belonging to the class $\mathscr{P}$.

\section{Theorem 3.1}

There exists a matrix $K$ and a sufficiently small positive $\varepsilon_{0}$ such that for all $0<\varepsilon \leqslant \varepsilon_{0}$ any perturbed system (5) with open-loop characteristic polynomial in $\mathscr{P}$ can be stabilized by a single controller (4) if and only if all coefficients $a_{1}^{j}, j=1, \ldots, m$, defined in (20), are positive.

The proof of Theorem 3.1, is based on the following lemmas:

\section{Lemma 3.1}

Consider a polynomial

$$
p(s)=\psi_{0} s^{n}+\psi_{1} s^{n-1}+\cdots+\psi_{n}
$$

where $n \geqslant 3, \psi_{0}=1, \psi_{i}>0, i=1, \ldots, n$. Let $\delta^{*}$ be the positive real solution of the equation $\delta(\delta+1)^{2}=1$. Then polynomial (21) is Hurwitz if the coefficients $\psi_{i}$ satisfy the condition

$$
\frac{\psi_{l-1} \psi_{l+2}}{\psi_{l} \psi_{l+1}} \leqslant \delta^{*}, \quad l=1, \ldots, n-2
$$

This lemma has been proved by Lipatov and Sokolov in Reference 9. Inequality (22) will henceforth be referred to as the Lipatov's condition.

\section{Lemma 3.2}

Consider the following four polynomials:

$$
\begin{aligned}
& p_{h 1}(s)=\gamma_{0} s^{n}+\gamma_{1}^{+} s^{n-1}+\gamma_{2}^{+} s^{n-2}+\gamma_{3}^{-} s^{n-3}+\gamma_{4}^{-} s^{n-4}+\cdots \\
& p_{h 2}(s)=\gamma_{0} s^{n}+\gamma_{1}^{-} s^{n-1}+\gamma_{2}^{-} s^{n-2}+\gamma_{3}^{+} s^{n-3}+\gamma_{4}^{+} s^{n-4}+\cdots \\
& p_{h 3}(s)=\gamma_{0} s^{n}+\gamma_{1}^{-} s^{n-1}+\gamma_{2}^{+} s^{n-2}+\gamma_{3}^{+} s^{n-3}+\gamma_{4}^{-} s^{n-4}+\cdots \\
& p_{h 4}(s)=\gamma_{0} s^{n}+\gamma_{1}^{+} s^{n-1}+\gamma_{2}^{-} s^{n-2}+\gamma_{3}^{-} s^{n-3}+\gamma_{4}^{+} s^{n-4}+\cdots
\end{aligned}
$$

where $n \geqslant 3, \gamma_{0}=1, \gamma_{i}^{-} \leqslant \gamma_{i}^{+}, 1 \leqslant i \leqslant n$ and $\gamma_{1}^{-}>0$. Let coefficients $\bar{a}_{i}, 1 \leqslant i \leqslant n$ be defined as

$$
\bar{a}_{i}(h)= \begin{cases}\gamma_{1}^{-} h^{-[(n-1)(i-1)] / 4+(i-1)^{2} / 4}-\gamma_{i}^{-} & \text {if } i \text { is odd } \\ h^{-[(n-1)(i+2)] / 4+i(i-2) / 4}-\gamma_{i}^{-} & \text {if } i \text { is even }\end{cases}
$$

where $h>0$. Then there exists a $h^{*}$ such that for all $0<h<h^{*}$ coefficient $\overline{a_{2}}$ is positive and the polynomials

$$
\begin{aligned}
& p_{h c l 1}(s)=\gamma_{0} s^{n}+\left(\gamma_{1}^{+}+\overline{a_{1}}(h)\right) s^{n-1}+\left(\gamma_{2}^{+}+\overline{a_{2}}(h)\right) s^{n-2}+\left(\gamma_{3}^{-}+\overline{a_{3}}(h)\right) s^{n-3}+\cdots \\
& p_{h c l 2}(s)=\gamma_{0} s^{n}+\left(\gamma_{1}^{-}+\overline{a_{1}}(h)\right) s^{n-1}+\left(\gamma_{2}^{-}+\overline{a_{2}}(h)\right) s^{n-2}+\left(\gamma_{3}^{+}+\overline{a_{3}}(h)\right) s^{n-3}+\cdots \\
& p_{h c l 3}(s)=\gamma_{0} s^{n}+\left(\gamma_{1}^{-}+\overline{a_{1}}(h)\right) s^{n-1}+\left(\gamma_{2}^{+}+\overline{a_{2}}(h)\right) s^{n-2}+\left(\gamma_{3}^{+}+\overline{a_{3}}(h)\right) s^{n-3}+\cdots \\
& p_{h c l 4}(s)=\gamma_{0} s^{n}+\left(\gamma_{1}^{+}+\overline{a_{1}}(h)\right) s^{n-1}+\left(\gamma_{2}^{-}+\overline{a_{2}}(h)\right) s^{n-2}+\left(\gamma_{3}^{-}+\overline{a_{3}}(h)\right) s^{n-3}+\cdots
\end{aligned}
$$

are Hurwitz. 
Proof. Let $\gamma_{i}^{j}$ denote the $i$ th coefficient, $1 \leqslant i \leqslant n$, of the $j$ th polynomial, $1 \leqslant j \leqslant 4$, in (23). First, we show that there exists $h^{*}$ such that for all $0<h \leqslant h^{*}$ polynomials (25) satisfy Lipatov's condition, i.e.

$$
\max _{1 \leqslant l \leqslant n-2,1 \leqslant j \leqslant 4} \frac{\left(\gamma_{l-1}^{j}+\overline{a_{l-1}}(h)\right)\left(\gamma_{l+2}^{j}+\overline{a_{l+2}}(h)\right)}{\left(\gamma_{l}^{j}+\overline{a_{l}}(h)\right)\left(\gamma_{l+1}^{j}+\overline{a_{l+1}}(h)\right)}<\delta^{*}
$$

where $\delta^{*}$ is defined in Lemma 3.1. Then, the statement of this lemma follows directly from Lemma 3.1. To show that Lipatov's condition holds, consider

$$
f_{l}(h) \triangleq \max _{1 \leqslant j \leqslant 4} \frac{\left(\gamma_{l-1}^{j}+\overline{a_{l-1}}(h)\right)\left(\gamma_{l+2}^{j}+\overline{a_{l+2}}(h)\right)}{\left(\gamma_{l}^{j}+\overline{a_{l}}(h)\right)\left(\gamma_{l+1}^{j}+\overline{a_{l+1}}(h)\right)}, \quad 1 \leqslant l \leqslant n-2
$$

It is easy to see that the function $f_{l}(h)$ is monotonically increasing with respect to $h$. Indeed, define $\overline{a_{0}}(h)=0 \quad$ and $\quad \gamma_{0}^{-}=\gamma_{0}^{+}=1 . \quad$ Let $\quad c_{q}^{+}(h)=\gamma_{q}^{+}+\overline{a_{q}}(h), \quad c_{q}^{-}(h)=\gamma_{q}^{-}+\overline{a_{q}}(h), \quad$ and $\Delta c_{q}=c_{q}^{+}(h)-c_{q}^{-}(h)=\gamma_{q}^{+}-\gamma_{q}^{-}$for $0 \leqslant q \leqslant n$. Then

$$
\begin{aligned}
f_{l}(h) & =\frac{c_{l-1}^{+}(h) c_{l+2}^{+}(h)}{c_{l}^{-}(h) c_{l+1}^{-}(h)}, \quad 1 \leqslant l \leqslant n-2 \\
& =\frac{c_{l-1}^{-}(h) c_{l+2}^{-}(h)}{c_{l}^{-}(h) c_{l+1}^{-}(h)}\left(1+\frac{\Delta c_{l-1}}{c_{l-1}^{-}(h)}\right)\left(1+\frac{\Delta c_{l+2}}{c_{l+2}^{-}(h)}\right) \\
& =\psi(h)\left(1+\frac{\Delta c_{l-1}}{c_{l-1}^{-}(h)}\right)\left(1+\frac{\Delta c_{l+2}}{c_{l+2}^{-}(h)}\right)
\end{aligned}
$$

where

$$
\psi(h)= \begin{cases}h^{(n+1) / 2} & \text { if } l=1 \\ h & \text { if } 2 \leqslant l \leqslant n-2\end{cases}
$$

We will show that each of the three factors in the right-hand side of (29) is a non-decreasing function of $h$. Indeed, $c_{0}^{-}=1, c_{1}^{-}=\gamma_{1}^{-}>0$, and since $c_{i}^{-}(h)=\gamma_{i}^{-}+\overline{a_{i}}(h)$ for $2 \leqslant i \leqslant n$, we observe from (24) that the exponent of $h$ in $c_{i}^{-}(h)$ is negative or zero. Also, $\Delta c_{q} \geqslant 0$ for $0 \leqslant q \leqslant n$ and from (30), $\psi\left(h_{1}\right)<\psi\left(h_{2}\right)$ for $0<h_{1}<h_{2}$. Thus, $f_{l}(h)$ is a continuous and monotonically increasing function of $h$. Also, as it follows from $(29), f_{l}(0)=0$, and $\lim _{h \rightarrow \infty} f_{l}(h)=\infty$. Therefore, by the Intermediate Value Theorem, there exists $h_{l}^{*}$ such that

$$
f_{l}\left(h_{l}^{*}\right)=\delta^{*}, \quad 1 \leqslant l \leqslant n-2
$$

Let $h^{*}=\min _{l} h_{l}^{*}$. Since $f_{l}(h)$ is monotonically increasing with $h$, it follows that $f_{l}\left(h^{*}\right) \leqslant f_{l}\left(h_{l}^{*}\right)$, $1 \leqslant l \leqslant n-2$. Therefore,

$$
\max _{l} f_{l}(h)=\max _{l, j} \frac{\left(\gamma_{l-1}^{j}+\overline{a_{l-1}}(h)\right)\left(\gamma_{l+2}^{j}+\overline{a_{l+2}}(h)\right)}{\left(\gamma_{l}^{j}+\overline{a_{l}}(h)\right)\left(\gamma_{l+1}^{j}+\overline{a_{l+1}}(h)\right)}<\max _{l} f_{l}\left(h^{*}\right) \leqslant \delta^{*}
$$

is satisfied for all $0<h<h^{*}$. Finally, to ensure that $\overline{a_{2}}>0$, i.e.

$$
h^{1-n}-\gamma_{2}^{-}>0
$$


choose $h$ in (24) as follows:

(i) if $\gamma_{2}^{-} \leqslant 0$, let $0<h<h^{*}$,

(ii) if $\gamma_{2}^{-}>0$, let $0<h<\min \left[\left(\gamma_{2}^{-}\right)^{1 /(1-n)}, h^{*}\right]$.

Proof of Theorem 3.1. Consider the closed-loop system (5), (4)

$$
\dot{x}=\left(A+\Delta A+B K f\left(\frac{t}{\varepsilon}\right)\right) x
$$

From (12), we obtain the following averaged equation:

$$
\dot{\bar{\zeta}}=\overline{\Phi^{-1}\left(\frac{t}{\varepsilon}\right)(A+\Delta A) \Phi\left(\frac{t}{\varepsilon}\right)} \bar{\zeta}
$$

where $\zeta$ is defined in (10).

According to References 5, 6 and 8 , if (34) is asymptotically stable, there exists a positive $\varepsilon_{0}$ sufficiently small such that for all $0<\varepsilon \leqslant \varepsilon_{0}$ equation (33) is also asymptotically stable. Note that $A+\Delta A$ and $B$ are in the controllable canonical form since the matrix $\Delta A$ affects only the last row of the system matrix $A$. The necessity part follows from the Jacob-Liouville theorem ${ }^{10}$ which states

$$
\operatorname{Tr}(A+\Delta A)=\operatorname{Tr} \overline{\left(\Phi^{-1}\left(\begin{array}{l}
t \\
\varepsilon
\end{array}\right)(A+\Delta A) \Phi\left(\begin{array}{l}
t \\
\varepsilon \\
\varepsilon
\end{array}\right)\right)}
$$

Since vibrational control cannot change the trace of the matrix $A+\Delta A$ in (33), it is necessary that $a_{1}^{j}>0, j=1, \ldots, m$ for (33) to be Hurwitz.

Sufficiency is based on Kharitonov's theorem ${ }^{11}$ and Lemma 3.2. Since $a_{1}^{j}>0, j=1, \ldots, m$ and $\overline{\phi(\tau)}=0$, we choose $k_{1}=0$. It then follows from (15) that the averaged equation (34) has the following characteristic polynomial:

$$
p_{c l}(s)=s^{n}+\left(a_{1}+\overline{a_{1}}\right) s^{n-1}+\left(a_{2}+\overline{a_{2}}\right) s^{n-2}+\cdots+\left(a_{n}+\overline{a_{n}}\right)
$$

where

$$
\overline{a_{i}}=k_{2} k_{i} \overline{\phi(t)^{2}}, \quad i=1, \ldots, n
$$

and the coefficients $a_{i}$ are the coefficients of the characteristic polynomial of the perturbed system (5). For $0<n \leqslant 2$, it is easy to construct $\overline{a_{i}}, 1 \leqslant i \leqslant n$ to stabilize (35). Hence, we will consider the case when $n \geqslant 3$. Let $\gamma_{i}^{-}=\min _{j} a_{i}^{j}, \gamma_{i}^{+}=\max _{j} a_{i}^{j}$, where $a_{i}^{j}$ denotes the $i$ th coefficient, $1 \leqslant i \leqslant n$, of the $j$ th vertex polynomial $1 \leqslant j \leqslant m$. We first construct four interval polynomials which contain the polynomial (35):

$$
\begin{aligned}
& p_{h c l 1}(s)=s^{n}+c_{1}^{+} s^{n-1}+c_{2}^{+} s^{n-2}+c_{3}^{-} s^{n-3}+c_{4}^{-} s^{n-4}+\cdots \\
& p_{h c l 2}(s)=s^{n}+c_{1}^{-} s^{n-1}+c_{2}^{-} s^{n-2}+c_{3}^{+} s^{n-3}+c_{4}^{+} s^{n-4}+\cdots \\
& p_{h c l 3}(s)=s^{n}+c_{1}^{-} s^{n-1}+c_{2}^{+} s^{n-2}+c_{3}^{+} s^{n-3}+c_{4}^{-} s^{n-4}+\cdots \\
& p_{h c l 4}(s)=s^{n}+c_{1}^{+} s^{n-1}+c_{2}^{-} s^{n-2}+c_{3}^{-} s^{n-3}+c_{4}^{+} s^{n-4}+\cdots
\end{aligned}
$$


where

$$
\begin{aligned}
& c_{i}^{-}=\gamma_{i}^{-}+\overline{a_{i}}, \quad 1 \leqslant i \leqslant n \\
& c_{i}^{+}=\gamma_{i}^{+}+\overline{a_{i}}
\end{aligned}
$$

Next, we need to determine $\overline{a_{i}}$ in (38) satisfying $\overline{a_{1}}=0, \overline{a_{2}}>0$ so that the four interal polynomials (37) are Hurwitz. It follows from Kharitonov's theorem that if the polynomials (37) are Hurwitz, then (35) is also Hurwitz. Choose $\overline{a_{i}}, 1 \leqslant i \leqslant n$ as defined in (24), then from Lemma 3.2, there exists a $h^{*}$ such that for all $0<h<h^{*}$, polynomials (37) are Hurwitz. The corresponding stabilizing state feedback gain $K$ can be computed from (36). As it follows References 5, 6 and 8, for each Hurwitz characteristic polynomial $p_{c l}(s)$ in (35) there exists $\varepsilon_{\mathscr{P}}>0$ such that for all $0<\varepsilon \leqslant \varepsilon_{\mathscr{P}}$ the corresponding closed-loop system of (33) is also asymptotically stable. In Reference 8 , a lower bound of $\varepsilon_{\mathscr{P}}$ was derived. This bound for $\varepsilon_{\mathscr{P}}$ is a continuous function of the coefficients of the open-loop characteristic polynomial $p(s)$. Since the set of open-loop characteristic polynomial $p(s) \in \mathscr{P}$ is closed and bounded, it follows from the property of continuous functions that a uniform lower bound of $\varepsilon_{\mathscr{P}}$ exists. The proof is completed by setting $\varepsilon_{0}=\min _{\mathscr{P}} \varepsilon_{\mathscr{P}}$.

\section{Remark 3.1}

The condition $a_{1}^{j}>0, j=1, \ldots, m$, in Theorem 3.1 is equivalent to the requirement that the trace of the perturbed matrix $A+\Delta A$ be negative.

\section{Remark 3.2}

Theorem 3.1 is an extension of the result obtained in Reference 12 for interval polynomials. The assumption that $\mathscr{P}$ is polytopic is weaker than Kharitonov's interval polynomial assumption because it allows for linearly dependent coefficient perturbations.

\section{Example 3.1}

Consider a 6th order system (5) with open-loop characteristic polynomial $p(s) \in \mathscr{P}$ where $\mathscr{P}$ is a polytope of polynomials (convex hull) with the following four vertex polynomials:

$$
\begin{aligned}
& p_{1}(s)=s^{6}+0 \cdot 5 s^{5}-0 \cdot 6 s^{4}+1 \cdot 5 s^{3}+2 \cdot 5 s^{2}+3 \cdot 4 s-3 \\
& p_{2}(s)=s^{6}+0 \cdot 7 s^{5}-s^{4}+2 s^{3}+2 s^{2}+4 s-1 \\
& p_{3}(s)=s^{6}+s^{5}+s^{4}+s^{3}+3 s^{2}+3 s-1 \\
& p_{4}(s)=s^{6}+0 \cdot 6 s^{5}+0 \cdot 2 s^{4}+1 \cdot 1 s^{3}+2 \cdot 9 s^{2}+3 \cdot 8 s+2
\end{aligned}
$$

This uncertain open-loop system is unstable since the vertex polynomials $p_{1}(s)$ and $p_{2}(s)$ are obviously unstable. With vibrational state feedback control (4), the characteristic polynomial (35) of the averaged equation (34) is bounded by the interval polynomials (37). Next, we construct $\overline{a_{i}}$, $1 \leqslant i \leqslant 6$, as defined in (24) and determine $h>0$ so that the interval polynomials (37) are 
Hurwitz. Following (28), we define the function $f_{l}(h)$ as

$$
f_{l}(h)=\frac{c_{i-1}^{+}(h) c_{i+2}^{+}(h)}{c_{i}^{-}(h) c_{i+1}^{-}(h)}, \quad 1 \leqslant l \leqslant 4
$$

and hence

$$
\begin{aligned}
& f_{1}(h)=h^{3 \cdot 5}\left(1+\frac{\Delta c_{3} h^{1 \cdot 5}}{\gamma_{1}^{-}}\right) \\
& f_{2}(h)=h\left(1+\frac{\Delta c_{1}}{\gamma_{1}^{-}}\right)\left(1+\Delta c_{4} h^{5 \cdot 5}\right) \\
& f_{3}(h)=h\left(1+\Delta c_{2} h^{5}\right)\left(1+\frac{\Delta c_{5} k}{\gamma_{1}^{-}}\right) \\
& f_{4}(h)=h\left(1+\frac{\Delta c_{3} h^{1 \cdot 5}}{\gamma_{1}^{-}}\right)\left(1+\Delta c_{6} h^{4}\right)
\end{aligned}
$$

where $\Delta c_{i}=c_{i}^{+}-c_{i}^{-}=\max _{j} a_{i}^{j}-\min _{j} a_{i}^{j}$. Consequently, with $\gamma_{1}^{-}=\min _{j} a_{1}^{j}=0 \cdot 5, \delta^{*}=0 \cdot 4656$, we compute $h_{l}^{*}, 1 \leqslant l \leqslant 4$, such that

$$
\begin{aligned}
& f_{1}\left(h_{1}^{*}\right)=\left(h_{1}^{*}\right)^{3 \cdot 5}\left(1+2\left(h_{1}^{*}\right)^{1 \cdot 5}\right)=\delta^{*} \\
& f_{2}\left(h_{2}^{*}\right)=h_{2}^{*}(1+1)\left(1+\left(h_{2}^{*}\right)^{5 \cdot 5}\right)=\delta^{*} \\
& f_{3}\left(h_{3}^{*}\right)=h_{3}^{*}\left(1+2\left(h_{3}^{*}\right)^{5}\right)\left(1+2 h_{3}^{*}\right)=\delta^{*} \\
& f_{4}\left(h_{4}^{*}\right)=h_{4}^{*}\left(1+2\left(h_{4}^{*}\right)^{1 \cdot 5}\right)\left(1+5\left(h_{4}^{*}\right)^{4}\right)=\delta^{*}
\end{aligned}
$$

Solving (39)-(42), we obtain $h_{1}^{*}=0.6536, \quad h_{2}^{*}=0.2327, \quad h_{3}^{*}=0.2925, h_{4}^{*}=0.3232$. Thus, $h^{*}=\min _{l} h_{1}^{*}=h_{2}^{*}=0 \cdot 2327$. Since $\min _{j} a_{2}^{j}<0$, for $0<h \leqslant 0 \cdot 2327$, the interval polynomials (37) and consequently, the characteristic polynomial (35) will be stabilized. Arbitrarily choose $h=0 \cdot 23$, then $\overline{a_{2}}=1554 \cdot 7, \overline{a_{3}}=3 \cdot 5, \overline{a_{4}}=3237 \cdot 6, \overline{a_{5}}=-0 \cdot 8$, and $\overline{a_{6}}=360 \cdot 3$. With $f(\tau)=\sin \tau$, the corresponding feedback gains in (4) are $k_{1}=0, k_{2}=55 \cdot 8, k_{3}=0 \cdot 1255, k_{4}=116 \cdot 1$, $k_{5}=-0 \cdot 0287$, and $k_{6}=12 \cdot 9$. From Theorem 2 of Reference 8 , the asymptotic stability of the averaged equation (34) ensures the asymptotic stability of the original system (33).

\section{CONCLUSIONS}

In this paper, we analysed the problem of stability robustness in closed-loop vibrational control. In particular, given a vibrational controller, we derived a bound on the unstructured uncertainty that preserves the stability of the closed-loop system. In addition, we addressed the question of existence of a single vibrational controller that stabilizes a set of plants. Here we showed that a vibrational controller, which stabilizes a polytope of characteristic polynomials, exists if and only if the sum of the eigenvalues of each vertex polynomial in the polytope is negative. The results obtained assure that practical applications of closed-loop vibrational control should not find particular impediments due to the lack of stability robustness. 


\section{REFERENCES}

1. Baillieul, J., 'Open-loop control using oscillatory inputs', Chap. 57.7, in The Control Handbook, W.S. Levine (Ed.), CRC Press, Boca Ratan, 1996.

2. Clinar, A., J. Deng, S. M. Meerkov and X. Shu, 'Vibrational control of an exothermic reaction in a CSTR: theory, simulations, experiments', A.I.C.L.E. J., 33, 353-365 (1987).

3. Kabamba, P. T., S. M. Meerkov and E.-K. Poh, 'Closed loop vibrational control state and output feedback stabilizability', Proc. 30th Conf. on Decision and Control, Brighton, U.K., December 1991.

4. Kabamba, P. T., S. M. Meerkov and E.-K. Poh, 'Closed loop vibrational control state and output feedback stabilizability and pole placement capabilities', Univ. of Mich. Contr. Group Report, No. CGR-92-17, August 1992.

5. Bogoliubov, N. N. and Y. A. Mitropolsky, Asymptotic Methods in the Theory of Nonlinear Oscillations, Gordon and Breach, New York, 1961.

6. Bellman, R., J. Bentsman, and S. M. Meerkov, 'Nonlinear system with fast parametric oscillations', J. Math. Anal. Appl., 97, 572 (1983).

7. Patel, R. V. and M. Toda, 'Quantitative measures of robustness for multivariable system', Proc. Joint Automat. Contr. Conf., paper TD8-A, 1980.

8. Bellman, R., J. Bentsman and S. M. Meerkov, 'Stability of fast periodic systems', IEEE Trans. Automat. Control, AC-31, 289-291 (1985).

9. Lipatov, A. V. and N. L. Sokolov, 'Some sufficient conditions for stability and instability of continous linear stationary systems', Automat. Remote Control, 39, 1285-1291 (1979).

10. Meerkov, S. M., 'Principle of vibrational control: theory and applications', IEEE Trans. Automat. Control, AC-25, 755-762 (1980).

11. Kharitonov, V. L., 'Asymptotic stability of an equilibrium position of a family of systems of linear differential equations', Differential'nye Uraveniya, 14(11) 1483-1485 (1978).

12. Yu, D., 'A necessary and sufficient condition for stabilizing a linear system with the characteristic polynomial as an interval polynomial by vibrational control', A Directed Study Report under the Supervision of S. M. Meerkov, Univ of Mich., June 1988. 